Supporting Information

\title{
Computational Design of Multisubstrate Enzyme Specificity
}

Antony D. St-Jacques, Marie-Ėve C. Eyahpaise \& Roberto A. Chica*

Department of Chemistry and Biomolecular Sciences, University of Ottawa, Ottawa, Ontario, Canada, K1N 6N5

* To whom correspondence should be addressed:

Roberto A. Chica, Email: rchica@uottawa.ca

Supporting Material included:

Supporting Information Text

Methods

Supporting Figures

Supporting Tables

Supporting References 


\section{Supporting Information Text}

Rationale for multichemical state analysis (MCSA). In order to design multisubstrate enzyme specificity, we need to compare energy values obtained for each sequence on multiple chemical states. This can be done using either a multistate design (MSD) or multistate analysis (MSA) framework. ${ }^{1}$ In MSD, individual single-state calculations in which rotamers for a specific aminoacid sequence are optimized on each state are run in parallel. Individual single-state energies are then combined into a single fitness value (e.g., arithmetic mean, sum, Boltzmann-weighted average, etc.) for each amino-acid sequence that represents its predicted stability across all states. MSD optimization algorithms ${ }^{2-3}$ attempt to improve this fitness value as a function of aminoacid sequence in order to find optimal sequences. In the context of this work, a total of 300 computer cores working in parallel ( 3 chemical states $\times 100$ backbone templates) would be required to redesign multisubstrate enzyme specificity using MSD. In addition to requiring significant computational resources, MSD necessitates the selection of an appropriate fitness function prior to calculation to ensure that the design objective will be achieved. This can be difficult to define a priori for complex design problems involving a large number of states.

Alternatively, MSA, which involves the post-CPD combination of energies obtained from independent single-state calculations into a single fitness value for each sequence, ${ }^{1}$ could be used for the design of multisubstrate enzyme specificity. Because MSA does not require that single-state calculations be run in parallel, computational resource requirements are significantly reduced. MSA also has the benefit of not requiring the assignment of a fitness function prior to rotamer-optimization calculations, increasing flexibility by allowing the user to evaluate various fitness functions post-CPD in order to identify the most appropriate one for achieving the design objective. However, there may not be any overlap between the lists of sequences that are obtained from each independent single-state calculation due to the stochasticity of the search algorithm, which would prevent comparison of sequence energies on every chemical state. While this issue can be solved by ensuring that every possible sequence is rotamer-optimized by each single-state calculation, it is not computationally tractable to do so when the searched sequence space is very large. To address these issues and enable the design of multisubstrate enzyme specificity using large structural ensembles, a new multistate CPD methodology, called multichemical state analysis (MCSA), was developed.

Contribution of chemical states to predictions. We investigated the effect of including multiple chemical states on design predictions. For this purpose, we generated probability tables for all combinations of chemical states and calculated the minimum size that would be required by our library design procedure to generate a combinatorial library containing each of the four highestactivity BCAT variants (Table S9). All libraries generated from alternate combinations of chemical states (HisGlu, HisPLP, and GluPLP) required larger minimum sizes to predict the four mutants. This was also the case for predictions made from individual chemical states, with the exception of the HIS state, which is sufficient to predict the four BCAT variants with minimum library sizes less than or equal to those obtained with the HisGluPLP state. This result suggests that it is the HIS state that drives selection of beneficial mutations in the HisGluPLP multichemical state. Indeed, all four point mutations comprised in the higher-activity variants (V109I, V109M, Y129F, G196C) rank in the top 2 mutations at their corresponding position on the HIS state, but only half or none do so on the GLU and PLP states, respectively (Figure 3). These results indicate that mutations that are beneficial for binding L-histidine have at least a neutral effect on L-glutamate binding and internal aldimine stability. Although these results demonstrate that consideration of only the HIS chemical state would have been sufficient to predict the four BCAT variants displaying enhanced L-histidine transamination activity reported here, the MCSA method is applicable to design problems requiring the consideration of two or more structurally distinct nonnative substrates. 


\section{Methods}

Structure preparation. The crystal structure of Escherichia coli BCAT in its 2-methyl-L-leucinebound external aldimine form was obtained from the Protein Data Bank (PDB ID: $1 \mathrm{ILL}^{4}$ ). Following removal of the 2-methyl group from the substrate analogue, coordinates for a single BCAT dimer were extracted to yield the structure of the L-leucine-bound external aldimine. This structure was then prepared using the following protocol in the Molecular Operating Environment (MOE) software. ${ }^{5}$ Hydrogens were added with the Protonate3D utility and manually adjusted to ensure that the protonation states of the pyridoxal 5'-phosphate cofactor (PLP) and the catalytic lysine residue (K159) were consistent with previous NMR studies ${ }^{6}$ and the aminotransferase catalytic mechanism. ${ }^{7}$ The resulting structure was then solvated in a rectangular box of water with counter ions $\left(\mathrm{Na}^{+}\right.$and $\left.\mathrm{Cl}^{-}\right)$under periodic boundary conditions with a box cut-off of $6 \AA$, and energyminimized by conjugate gradient energy minimization to a root mean square gradient $<0.1 \mathrm{kcal}$ $\mathrm{mol}^{-1} \AA^{-1}$ using the AMBER99 force field ${ }^{8}$ with a combined explicit solvent and implicit reaction field solvent model set up using the MOE software package. The BCAT internal aldimine structure was prepared using a similar protocol with the exception that the input dimer structure was constructed by first deleting the coordinates of the 2-methyl-L-leucine analogue and then replacing the coordinates of the PLP cofactor and Y164, Y31, and K159 active-site residues by those from the $1 / 1 \mathrm{~K}$ crystal structure ${ }^{4}$ (all-atom root mean square deviation to $1 / 1 \mathrm{~L}=0.15 \AA$ ). Although the $1 \mathrm{I} 1 \mathrm{~K}$ structure is that of the BCAT internal aldimine, this structure was not suitable for our calculations because it lacks electron density for the interdomain loop (residues 125-136) that hangs over the active-site entrance.

Ensemble generation. All molecular dynamics simulations were run using NAMD $^{9}$ version 2.9 with the AMBER99 ${ }^{8}$ force field, and a combined explicit solvent and implicit reaction-field solvent model prepared using MOE. ${ }^{5}$ The minimized BCAT internal and external aldimine structures in rectangular boxes of water described above were heated to $300 \mathrm{~K}$ over $10 \mathrm{ps}$ and equilibrated for an additional $10 \mathrm{ps}$ at constant pressure (NPT). Following equilibration, a production run of $1 \mathrm{~ns}$ at constant pressure was performed. From each trajectory, 100 structures were extracted at $10-$ ps intervals and energy-minimized as described above to a root mean square gradient $<7 \mathrm{kcal}$ $\mathrm{mol}^{-1} \AA^{-1}$. The resulting 100-member ensembles derived from the BCAT internal and external aldimine structures had a diversity (i.e. average backbone root mean square deviation between members of the ensemble) of $0.8 \pm 0.2 \AA$ and $0.8 \pm 0.1 \AA$, respectively.

Substrate rotamers. Coordinates of the PLP-bound L-leucine aldimine ligand were extracted from the minimized BCAT external aldimine structure described above. Following replacement of the L-leucine side chain by that of L-histidine (both tautomers), L-glutamate, L-leucine, or Lthreonine, rotamers for these substrates were generated by rotating $X$ dihedral angles to the following values: $+60,180$, and -60 degrees, with a deviation of \pm 10 and 20 degrees for $\mathrm{sp}^{3}-\mathrm{sp}^{3}$ bonds, or $0,+60,+120,180,-120$, and -60 degrees, with a deviation of \pm 10 and 20 degrees for $\mathrm{sp}^{3}-\mathrm{sp}^{2}$ bonds. Then, the internal energy of each rotamer was calculated in vacuo using MOE with the previously described force field. The inclusion of PLP in the internal energy calculations allowed for the rejection of substrate rotamers that would clash with cofactor atoms. Next, the Boltzmann probability of each side-chain rotamer was calculated using Equation 1, where, for a given rotamer, $i, P_{i}$ is the probability, $E_{i}$ is the internal energy, $k_{B}$ is the Boltzmann constant, $T$ is the temperature $(300 \mathrm{~K})$, and $n$ is the number of all rotamers evaluated. The top $95 \%$ rotamers $^{10}$ ranked by probability for each substrate were included in the corresponding substrate rotamer library (738, 291, 45, and 28 rotamers for L-glutamate, L-histidine, L-leucine, and L-threonine, respectively). 


$$
P_{i}=\frac{e^{-E_{i} / k_{B} T}}{\sum_{i=1}^{n} e^{-E_{i} / k_{B} T}}
$$

Equation 1. Boltzmann probability.

MCSA. All calculations were performed using the PHOENIX protein design software ${ }^{11-12}$ with the fast and accurate side-chain topology and energy refinement (FASTER) algorithm ${ }^{13}$ for sequence optimization. The backbone-independent Dunbrack rotamer library ${ }^{14}$ with expansions of \pm 1 standard deviation around $X_{1}$ and $X_{2}$ was used to provide side-chain conformations of BCAT residues to be threaded onto each fixed backbone template. Rotamers for the L-histidine, Lglutamate, L-leucine, and L-threonine substrate side chains prepared as described above were threaded on the PLP cofactor to replace the L-leucine substrate on each backbone comprised in the ensemble. Side-chain rotamers of BCAT residues were optimized on each template structure using all proteinogenic amino acids with the exception of proline and lysine. Sequences were scored using a five-term potential energy function consisting of a Lennard-Jones 12-6 van der Waals term from the Dreiding II force field ${ }^{15}$ with atomic radii scaled by 0.9 , a direction-dependent hydrogen bond term with a well depth of $8.0 \mathrm{kcal} \mathrm{mol}^{-1}$ and an equilibrium donor-acceptor distance of $2.8 \AA,{ }^{16}$ an electrostatic energy term modelled using Coulomb's law with a distance-dependent dielectric of 10 , an occlusion-based solvation penalty term, ${ }^{12,16-17}$ and a secondary structural propensity term. ${ }^{18}$

CPD calculations were performed using the procedure shown on Figure S5. Briefly, for each individual chemical state represented by a 100-member backbone ensemble (Figure S5a), the six design positions $(31,109,126,129,196$, and 258) were separated into three subdesigns (Figure S5b) so that the total number of sequences that were to be rotamer-optimized could be reduced to a value that was computationally tractable (Table S1). Importantly, each subdesign must contain at least one design position in common with each of the other subdesigns to ensure that results from each subdesign can be recombined into a single set of mutations predicted to satisfy the design objective. Positions in common were selected because of their close spatial proximity in the BCAT active site to at least two sets of positions, each set being unique to a different subdesign. This is done to maximize the number of strong two-body interactions in the BCAT active site that are evaluated during design. For every subdesign, rotamers were optimized in 100 independent single-state design calculations, yielding a ranked list of scored sequences for each template included in the backbone ensemble (Figure S5c). Sequence energies obtained from each single-state calculation were combined post-CPD using a Boltzmann-weighted average at $300 \mathrm{~K}$ of the individual energies for each backbone across the 100-member ensemble, and re-ranked based on their Boltzmann-weighted average energies to generate a new ranked list of scored sequences for each subdesign (Figure S5d). For the multichemical states (e.g., HisGluPLP, HisGlu, GluPLP, HisPLP, etc.), energies of corresponding subdesign sequences from each chemical state were averaged (arithmetic mean) and sequences re-ranked (Figure S5e). These sequence lists were used to construct a position energy matrix (PEM) for each subdesign (Figure S5f) as described below. Subdesign PEMs were combined into a global PEM (Figure S5g) representing the energy of each amino acid substitution at every design position for every individual or multichemical state, by using the elementwise mean. Using this procedure, the largest subdesign calculation (104,976 or $18^{4}$ sequences) took approximately two weeks on an individual Intel Xeon $2.7 \mathrm{GHz}$ processor, and one processor was used per ensemble member.

Construction of position matrices. From the energies computed by multistate analysis for each subdesign, the Boltzmann weight $(w)$ was calculated for each sequence using Equation 2, where $E$ is the energy of the sequence, $k_{B}$ is the Boltzmann constant, and $T$ is the temperature $(300 \mathrm{~K})$. With these Boltzmann weights for all sequences $(S)$, a cumulative partition function $(q)$ for every 
amino acid (aa) at each position ( $n$ ) was computed using Equation 3, where the Boltzmann weight of a sequence is only added to $q_{a a, n}$ if the identity of the residue at position $n\left(r e s i_{n}\right)$ is aa. The PEM was constructed by using Equation 4 to convert the cumulative partition function of amino acid aa at position $n$ to energy ( $300 \mathrm{~K})$. The Position Probability Matrix (PPM) was constructed by first calculating a table of cumulative partition functions from the global PEM using Equation 4 and subsequently dividing each partition function by the sum of partition functions for all amino acids at that position, $Q_{n}$. Scripts for the generation of position matrices are provided as Supporting Information files.

$$
w=e^{-E / k_{B} T}
$$

Equation 2. The Boltzmann weight.

$$
q_{a a, n}=\sum_{i=1}^{S} w_{i}\left[r e s i_{n}=a a\right]
$$

Equation 3. The cumulative partition function.

$$
E=-k_{B} T \cdot \ln \left(q_{a a, n}\right)
$$

Equation 4. The Boltzmann energy.

$$
P P M=\left[\begin{array}{ccccc}
q_{A, 1} & q_{A, 2} & \ldots & q_{A, n-1} & q_{A, n} \\
q_{C, 1} & q_{C, 2} & \ldots & q_{C, n-1} & q_{C, n} \\
\vdots & \vdots & \ddots & \vdots & \vdots \\
q_{W, 1} & q_{W, 2} & \ldots & q_{W, n-1} & q_{W, n} \\
q_{Y, 1} & q_{Y, 2} & \ldots & q_{Y, n-1} & q_{Y, n}
\end{array}\right] \div\left[\begin{array}{c}
Q_{1} \\
Q_{2} \\
\vdots \\
Q_{n-1} \\
Q_{n}
\end{array}\right]=\left[\begin{array}{ccccc}
p_{A, 1} & p_{A, 2} & \ldots & p_{A, n-1} & p_{A, n} \\
p_{C, 1} & p_{C, 2} & \ldots & p_{C, n-1} & p_{C, n} \\
\vdots & \vdots & \ddots & \vdots & \vdots \\
p_{W, 1} & p_{W, 2} & \ldots & p_{W, n-1} & p_{W, n} \\
& & & & \\
p_{Y, 1} & p_{Y, 2} & \ldots & p_{Y, n-1} & p_{Y, n}
\end{array}\right]
$$

Equation 5. Position Probability Matrix.

Library design. Library design was performed with the CLEARSS algorithm ${ }^{19}$ implemented in PHOENIX using the PPM described above as input. For a specific library size configuration, which is the specific number of amino acids at each position in the protein (e.g., 4 amino acids at position 1, 3 amino acids at position 2, etc.), the wild-type residue and the highest probability set of amino acids at each position was included in the library. To identify the optimal library size configuration, all configurations that lead to a combinatorial library of a target size were scored by taking the sum of all partition functions of the chosen amino acid sets over all positions, and the highest scoring library was selected. A script for running the CLEARSS algorithm on the PPM is provided as a Supporting Information file.

Chemicals and enzymes. All reagents used were of the highest available purity. Synthetic oligonucleotides were purchased from Eurofins MWG Operon. Restriction enzymes and DNAmodifying enzymes were purchased from New England Biolabs. Ni-NTA agarose resin was 
purchased from Bio-Rad Laboratories. All aqueous solutions were prepared using water purified with a Barnstead Nanopure Diamond system.

Mutant library construction. The codon-optimized ( $E$. coli) and his-tagged (N-terminus) gene of E. coli BCAT (Uniprot ID: POAB80) was purchased from Integrated DNA Technologies and subcloned into the pET-11a expression vector (Novagen) via the $\mathrm{Ndel} / \mathrm{BamHI}$ restriction sites. The resulting plasmid was transformed into chemically-competent $E$. coli BL21-Gold(DE3) cells (Agilent). Mutations were introduced into the BCAT gene by overlap extension mutagenesis ${ }^{20}$ using VentR DNA Polymerase. Briefly, external primers were used in combination with sets of complementary pairs of oligonucleotides containing the mutated codons in individual polymerase chain reactions (PCRs). The resulting overlapping fragments were gel-purified (Omega Biotek) and recombined by overlap extension PCR. The resulting amplicons were digested with Ndel/BamHI, gel-purified, and ligated into pET-11a expression vector with T4 DNA ligase. Constructs were verified by sequencing the entire open reading frame, and were transformed into chemically-competent $E$. coli BL21-Gold(DE3) cells (Agilent) for protein expression.

Preparation of clarified cell lysates. The DNA libraries prepared as described above were transformed into chemically competent E. coli BL21-Gold(DE3) cells (Agilent). Colonies were picked into individual wells of V96 MicroWell polypropylene plates (Nunc) containing $300 \mu \mathrm{L}$ of medium (LB with $100 \mu \mathrm{g} / \mathrm{mL}$ ampicillin supplemented with $10 \%$ glycerol). The plates were covered with a sterile breathable rayon membrane (VWR) and incubated overnight at $37^{\circ} \mathrm{C}$ with shaking. After incubation, these mother plates were used to inoculate sterile Nunc V96 MicroWell polypropylene plates ("daughter" plates) containing $300 \mu \mathrm{L}$ of Overnight Express Instant TB media (Novagen) supplemented with ampicillin per well. Daughter plates were sealed with breathable membranes and incubated overnight $\left(37^{\circ} \mathrm{C}, 250 \mathrm{rpm}\right)$. After incubation, the cells were harvested by centrifugation $\left(3000 \mathrm{~g}, 30 \mathrm{~min}, 4^{\circ} \mathrm{C}\right.$ ) and the cell pellets were washed twice with phosphate-buffered saline ( $\mathrm{pH} 7.4)$. Washed cell pellets were resuspended in lysis buffer (100 $\mathrm{mM}$ potassium phosphate buffer [pH 8.0] containing 1× Bug Buster Protein Extraction Reagent [Novagen], $5 \mathrm{U} / \mathrm{mL}$ Benzonase Nuclease [EMD], and $1 \mathrm{mg} / \mathrm{mL}$ lysozyme). The clarified lysate was collected following centrifugation and stored at $4{ }^{\circ} \mathrm{C}$ until used in the enzymatic assay.

Library screening. All assays were performed in $200-\mu \mathrm{L}$ reactions at $37^{\circ} \mathrm{C}$ in $100 \mathrm{mM}$ potassium phosphate buffer ( $\mathrm{pH} 8.0)$. The standard reaction mixture contained final concentrations of $16 \mu \mathrm{M}$ pyridoxal phosphate (PLP), $2 \mathrm{mM}$ L-histidine, $0.5 \mathrm{mM}$ a-ketoglutarate, $1 \mathrm{U}$ of glutamate dehydrogenase $(\mathrm{GDH})$ from bovine liver (Sigma), and $5 \mathrm{mM} \mathrm{NAD}$. Plates containing the standard reaction mixture were preincubated at $37^{\circ} \mathrm{C}$ for $5 \mathrm{~min}$ prior to initiation of the reaction by the addition of $20 \mu \mathrm{L}$ of clarified cell lysates prepared as described earlier. Enzyme reactions were monitored by measuring absorbance of NADH at $340 \mathrm{~nm}\left(\varepsilon=6220 \mathrm{M}^{-1} \mathrm{~cm}^{-1}\right)$ every 12 sec for 30 or $60 \mathrm{~min}$ in individual wells of 96-well plates (Greiner Bio-One) using a SpectraMax 384 Plus plate reader (Molecular Devices).

Protein expression and purification. Selected mutants were expressed and purified as described by Mironov et al. ${ }^{21}$ Briefly, expression vectors containing the aminotransferase genes were transformed into $E$. coli BL21-Gold(DE3) cells. The transformed cells were grown in $500 \mathrm{~mL}$ Luria-Bertani medium containing $100 \mu \mathrm{g} / \mathrm{mL}$ ampicillin at $37^{\circ} \mathrm{C}$ until they reached an OD600 of 0.6. Isopropyl $\beta$-D-1-thiogalactopyranoside $(1 \mathrm{mM})$ was added to the flasks to induce protein expression, followed by shaking for an additional $3 \mathrm{~h}$ at $37{ }^{\circ} \mathrm{C}$. Cells were harvested by centrifugation and then lysed using an EmulsiFlex-B15 cell disruptor (Avestin). Proteins were then extracted and purified by immobilized metal affinity chromatography, according to manufacturer's protocol. Elution fractions containing the aminotransferases were desalted using Econo-Pac 10DG columns (Bio-Rad). Protein concentrations were quantified using a modified version of the 
Bradford assay, where the calibration curve is constructed as a plot of the ratio of the absorbance measurements at 590 and $450 \mathrm{~nm}$ versus concentration. ${ }^{22}$

Steady-state kinetics. To measure steady-state kinetics for $\alpha$-ketoglutarate, assays were performed by varying the a-ketoglutarate concentration up to $10 \mathrm{mM}$ in the presence of $2 \mathrm{mM}$ L-leucine, $5 \mathrm{mM} \mathrm{NAD}^{+}, 16 \mu \mathrm{M}$ PLP, $1 \mathrm{U} \mathrm{GDH}$, and approximately $10 \mathrm{mU}$ of aminotransferase in $100 \mathrm{mM}$ potassium phosphate buffer $\left(\mathrm{pH} 8,37^{\circ} \mathrm{C}\right)$. To measure steady-state kinetics for L-histidine, L-leucine, and L-threonine, assays were performed by varying the amino-acid concentration up to $86 \mathrm{mM}$ in the presence of 1.25 or $10 \mathrm{mM}$--ketoglutarate, $5 \mathrm{mM} \mathrm{NAD}^{+}, 16 \mu \mathrm{M}$ PLP, $1 \mathrm{U} \mathrm{GDH}$, and approximately $10 \mathrm{mU}$ of aminotransferase in $100 \mathrm{mM}$ potassium phosphate buffer $\left(\mathrm{pH} 8,37^{\circ} \mathrm{C}\right)$. The $\mathrm{pH}$ of all reaction mixtures were adjusted to 8.0 prior to initiation of the assay. Linear phases of the kinetic traces were used to measure initial reaction rates. Initial reaction rates at different amino-acid concentrations were fit to the Michaelis-Menten equation using Python ver.2.7.15 with the scipy.optimize.curve_fit function (scipy ver.1.1.0). For mutants displaying substrate inhibition, fitting of the kinetic data was done with a rate equation that takes into account this type of inhibition: $v_{0}=\left(v_{\max }[S]\right) /\left(K_{M}+[S]+[S]^{2} / K_{i}\right)$. 


\section{Supporting Figures}
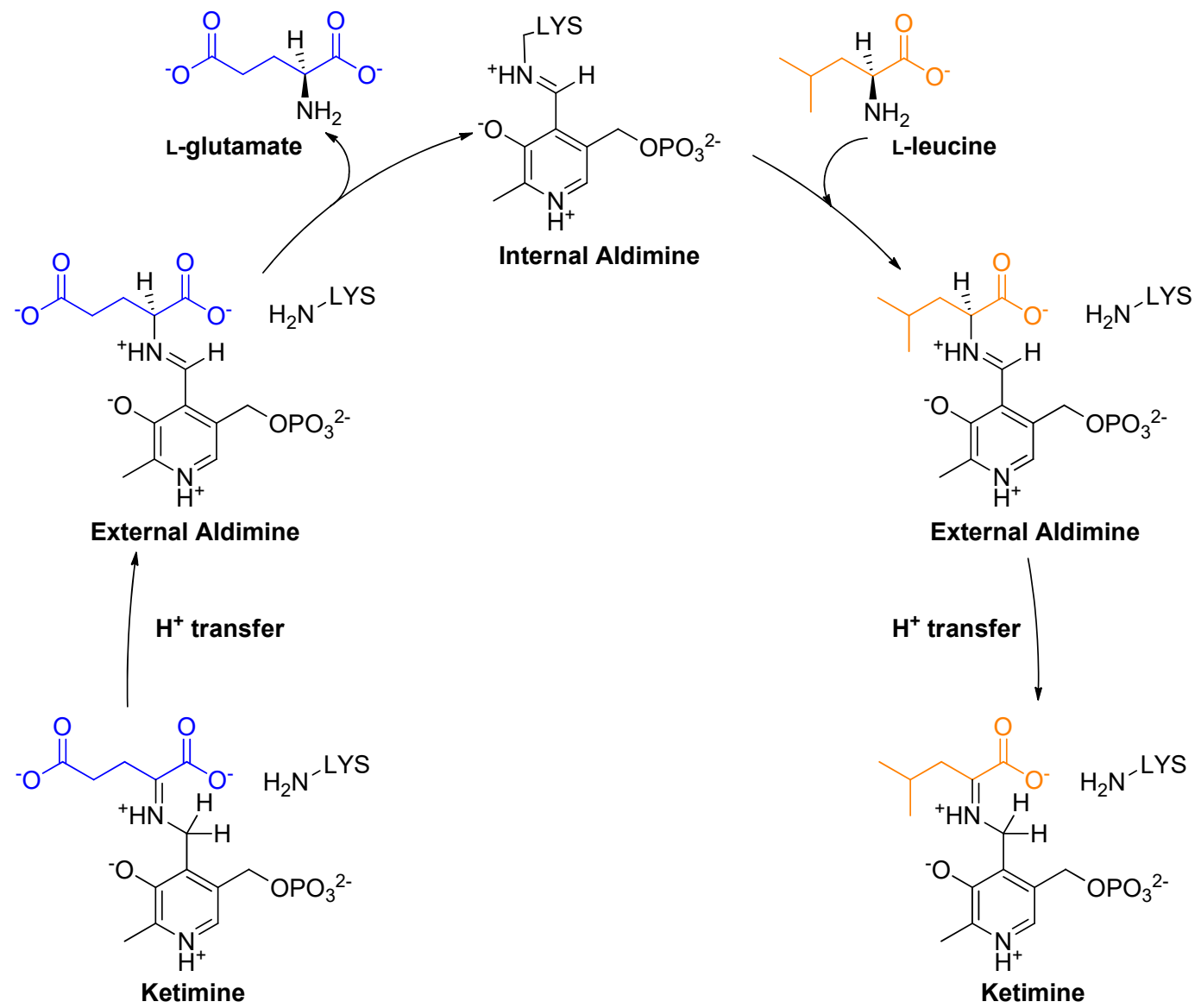

$$
\text { External Aldimine }
$$
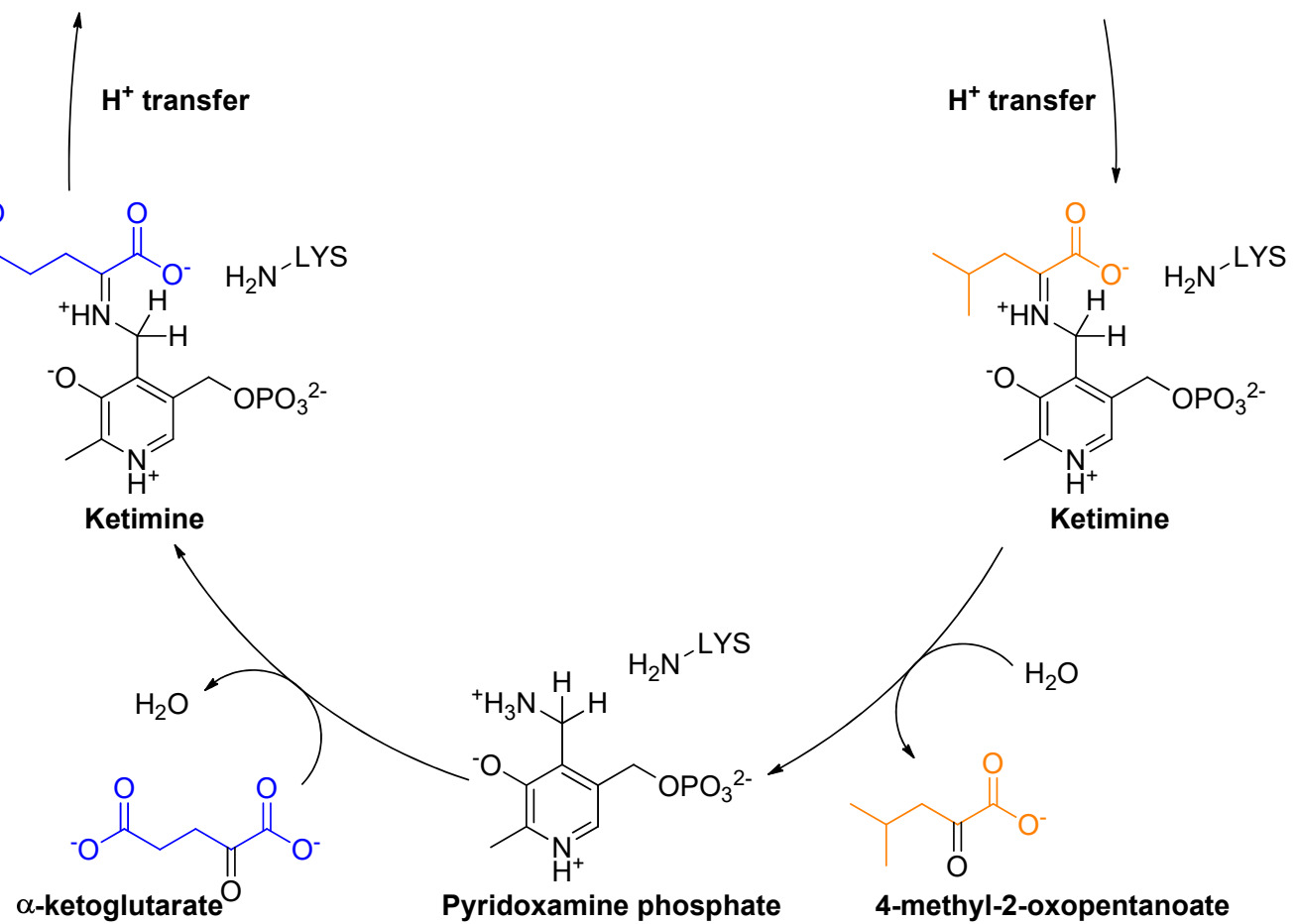

Slutarate

Pyridoxamine phosphate

Figure S1. Transamination of L-leucine with $\alpha$-ketoglutarate catalyzed by BCAT. This reaction proceeds via a bi bi ping-pong mechanism. 

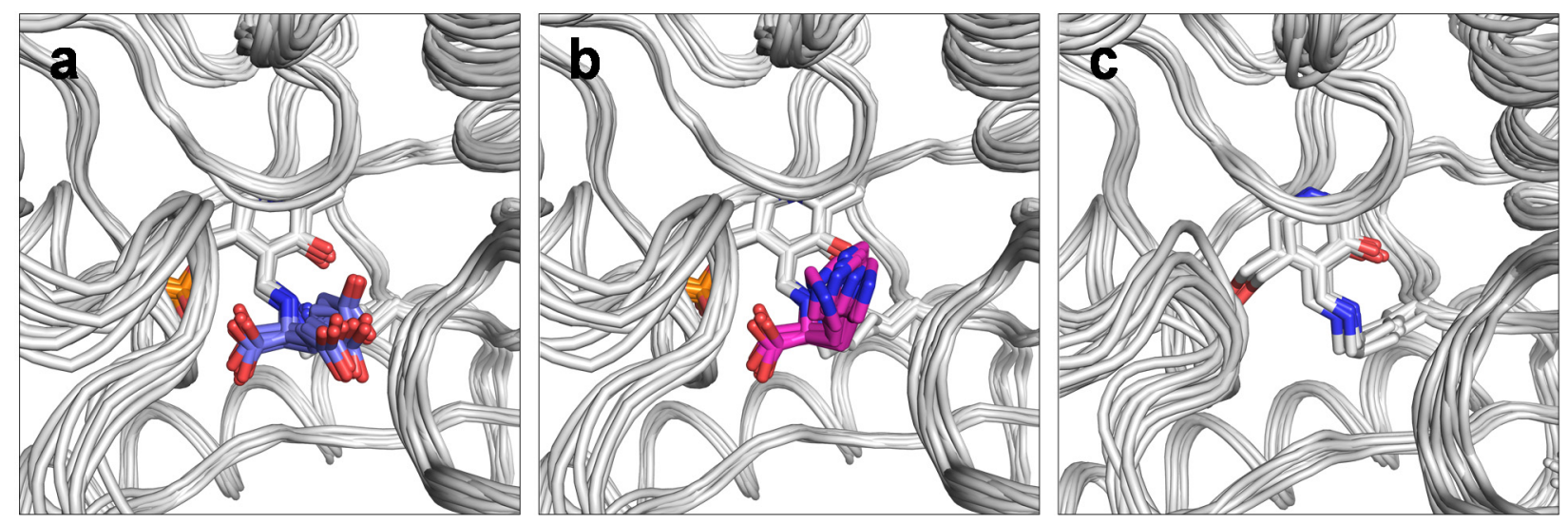

Figure S2. Backbone ensembles used in MCSA calculations. The (a) GLU, (b) HIS, and (c) PLP states were each modeled as a 100-member backbone ensemble to approximate protein flexibility. For clarity, only 10 out of 100 templates are shown for each ensemble. The L-glutamate and L-histidine substrates, and the PLP cofactor, are shown as blue, magenta, or white sticks, respectively. 


\section{a}
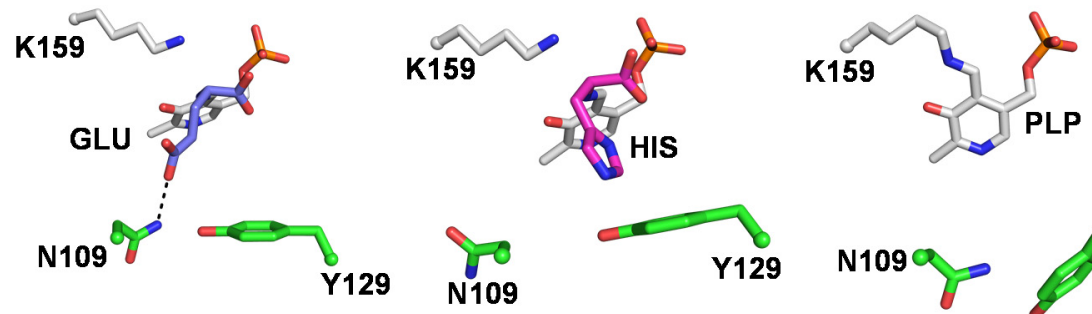

b
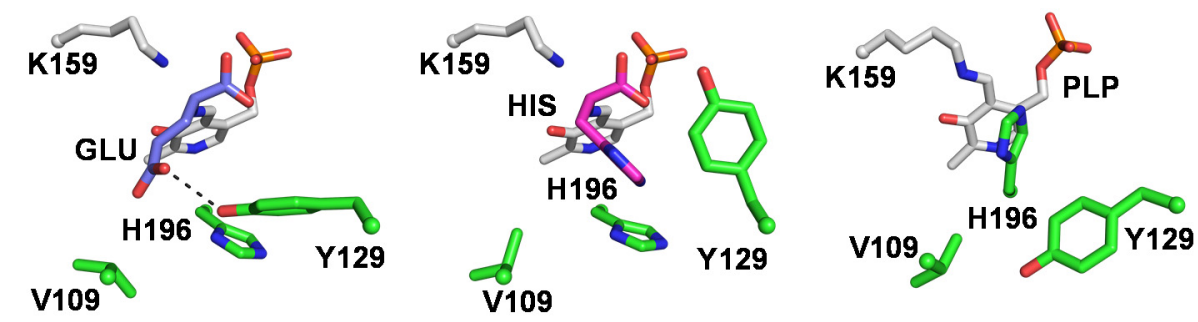

Figure S3. Structural models of select BCAT sequences designed on the GLU, HIS, and PLP chemical states. (a) V109N variant, (b) G196H variant. The PLP cofactor and catalytic lysine residue are colored white. The L-glutamate and L-histidine substrates are colored blue and magenta, respectively. Active-site residues are colored green, and their $\mathrm{C}_{\alpha}$ atom is shown as a sphere. $\mathrm{H}$-bonds are shown as dashed lines. 

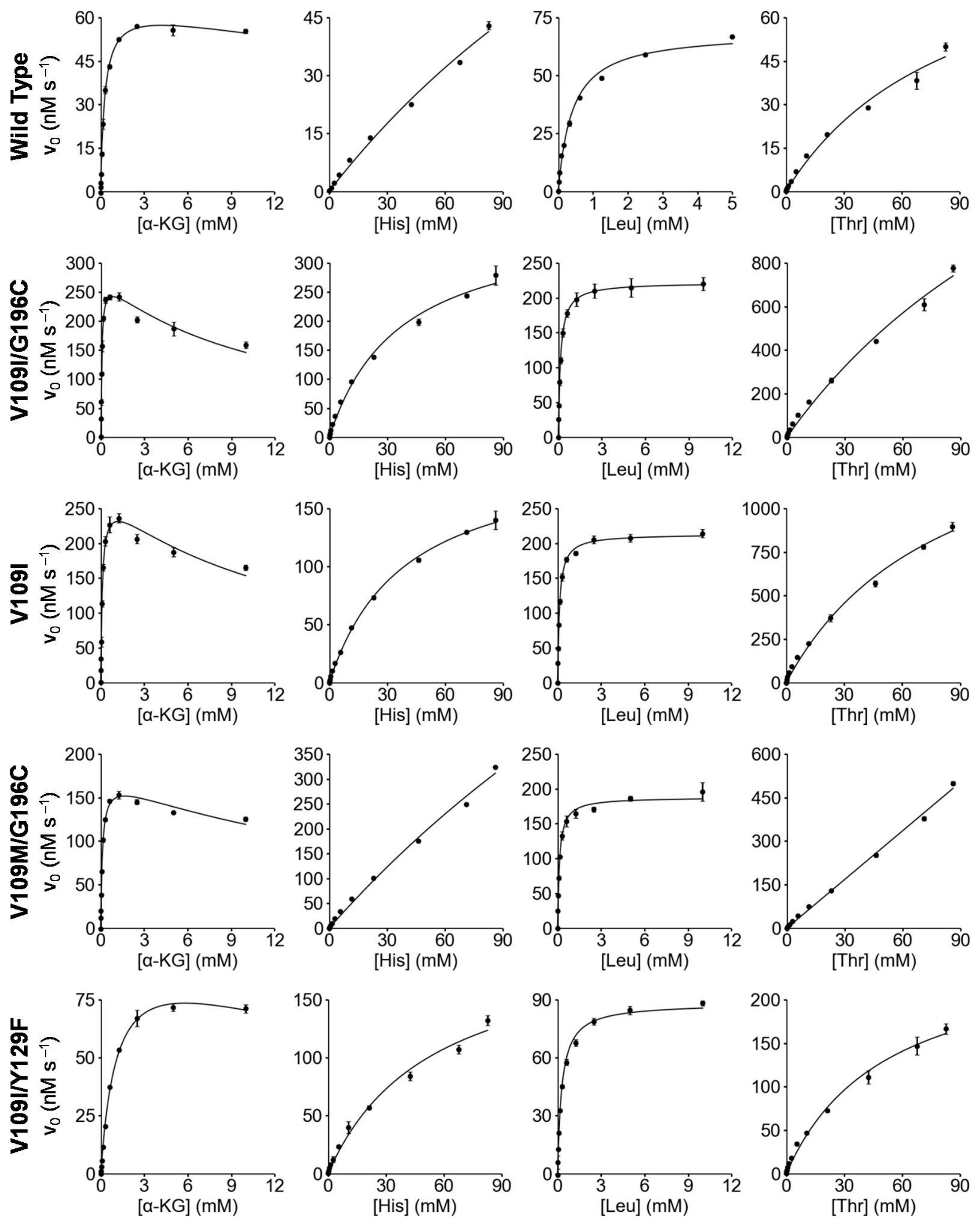

Figure S4. Steady-state kinetics of E. coli BCAT and its mutants. Michaelis-Menten plots of initial rates as a function of substrate concentrations are shown. All experiments were performed in triplicate. 
a

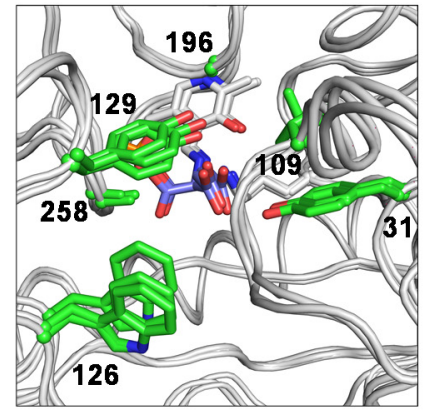

cLU state

b

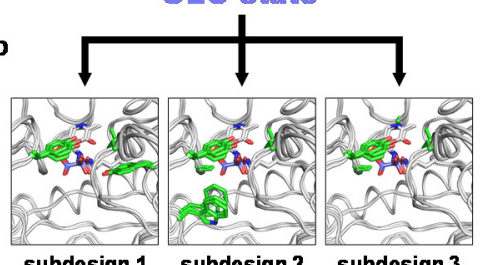

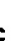
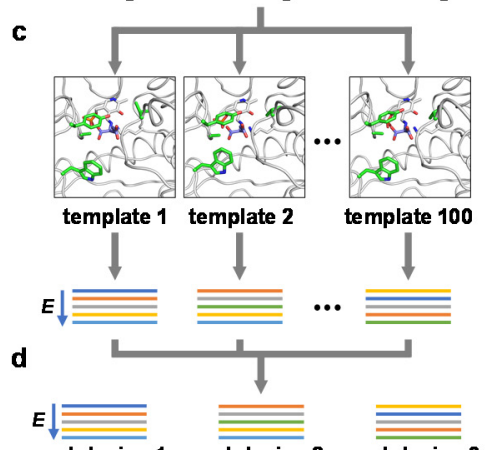

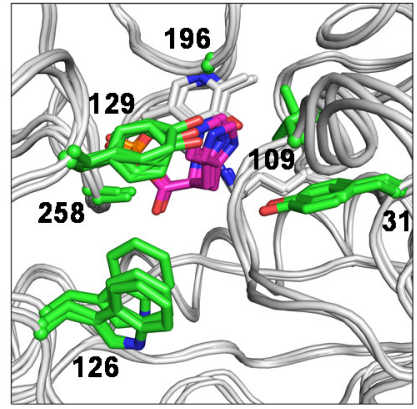

HIS state
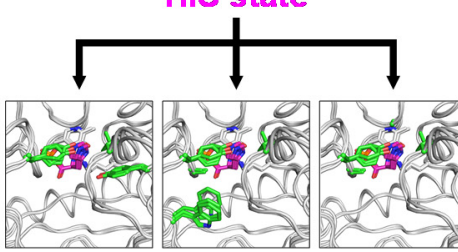

subdesign 1 subdesign 2 subdesign 3

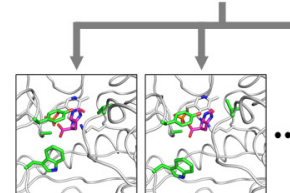

template 1 template 2 template 100

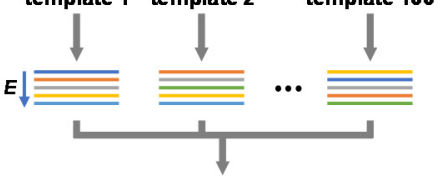

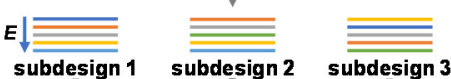

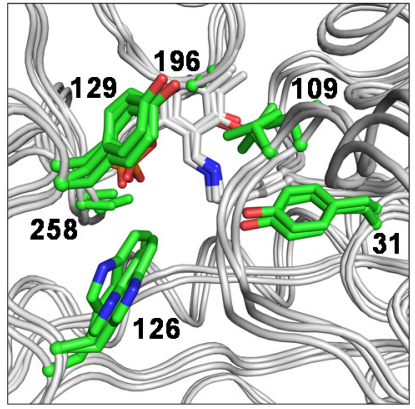

PLP state
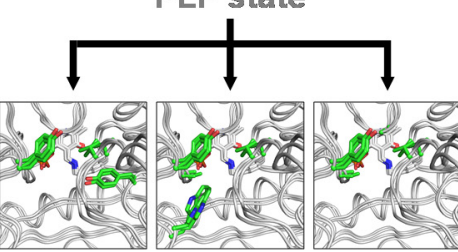

subdesign 1 subdesign 2 subdesign 3

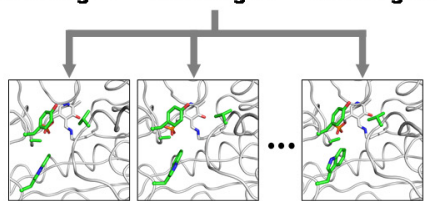

template 1 template 2 template 100
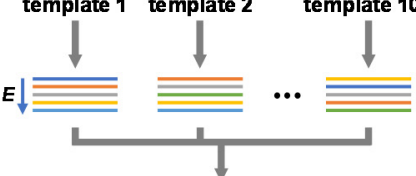

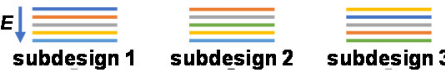

e

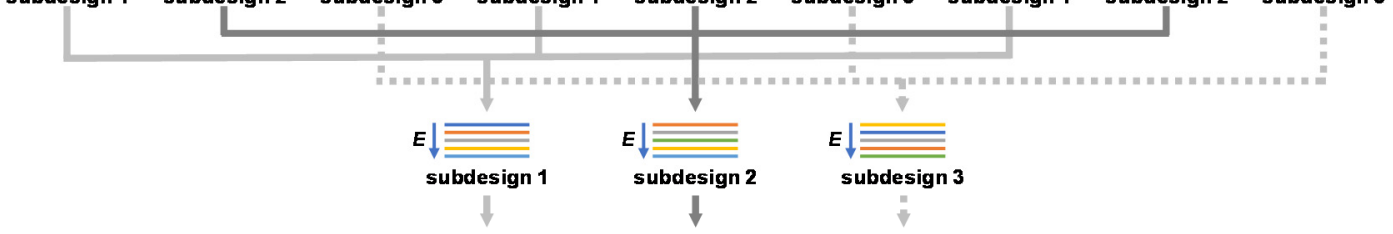

f

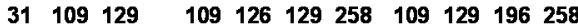

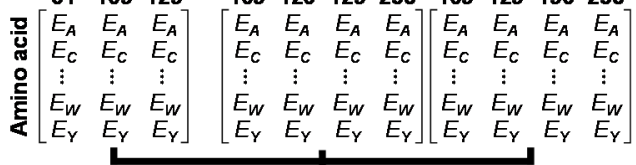

g

$$
\begin{aligned}
& 31109126129196258 \\
& \text { 흔 }\left[\begin{array}{llllll}
E_{A} & E_{A} & E_{A} & E_{A} & E_{A} & E_{A} \\
E_{C} & E_{C} & E_{C} & E_{C} & E_{C} & E_{C}
\end{array}\right]
\end{aligned}
$$

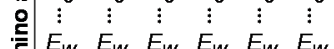

$$
\begin{aligned}
& \text { है। }
\end{aligned}
$$

Figure S5. Redesign of BCAT multisubstrate specificity using MCSA. For detailed description, see Methods. (a) Amino acids were designed at six positions $(31,109,126,129,196,258)$ on three chemical states (HIS, GLU, PLP), each one represented by a 100-member backbone ensemble. For clarity, only three backbone templates are shown. (b) Design positions were separated into three subdesigns. (c) Rotamer optimization was performed on each template using independent single-state design calculations, which yielded a ranked list of scored sequences (shown as colored lines) for each template. (d) Sequence energies $(E)$ obtained from each single-state calculation were combined using the Boltzmann-weighted average, and re-ranked to generate a list of scored sequences for each subdesign. (e) Sequence energies from corresponding subdesigns on individual chemical states were combined using the arithmetic mean. (f) Position energy matrices (PEMs) for each subdesign were generated. (g) Subdesign PEMs were combined into a global PEM using the elementwise mean. 


\section{Supporting Tables}

Table S1. BCAT residues optimized by computational protein design

\begin{tabular}{llll}
\hline Subdesign & Designed positions $^{a}$ & Floated positions $^{b}$ & Total sequences \\
\hline $\mathbf{1}$ & Y31, V109, Y129 & R97, W126, E197 & $5,832\left(18^{3}\right)$ \\
$\mathbf{2}$ & V109, W126, Y129, A258 & R97, F124, E197 & $104,976\left(18^{4}\right)$ \\
$\mathbf{3}$ & V109, Y129, G196, A258 & Y31, R97, W126, E197 & $104,976\left(18^{4}\right)$ \\
\hline
\end{tabular}

${ }^{a}$ All proteinogenic amino acids with the exception of proline and lysine were allowed at these positions.

${ }^{b}$ Residues whose rotameric configuration but not identity was allowed to vary. This also includes the substrates. 
Table S2. Position probability matrix of HIS chemical state

\begin{tabular}{|c|c|c|c|c|c|c|}
\hline & $\mathbf{3 1}$ & $\mathbf{1 0 9}$ & $\mathbf{1 2 6}$ & $\mathbf{1 2 9}$ & $\mathbf{1 9 6}$ & $\mathbf{2 5 8}$ \\
\hline $\mathbf{A}$ & $<0.1$ & 0.2 & $<0.1$ & $<0.1$ & 5.9 & $<0.1$ \\
\hline $\mathbf{C}$ & $<0.1$ & 2 & $<0.1$ & $<0.1$ & 77.2 & $<0.1$ \\
\hline $\mathbf{D}$ & $<0.1$ & $<0.1$ & $<0.1$ & $<0.1$ & $<0.1$ & $<0.1$ \\
\hline $\mathbf{E}$ & $<0.1$ & $<0.1$ & $<0.1$ & $<0.1$ & $<0.1$ & $<0.1$ \\
\hline $\mathbf{F}$ & 34.8 & $<0.1$ & $<0.1$ & 99.2 & $<0.1$ & $<0.1$ \\
\hline $\mathbf{G}$ & $<0.1$ & $<0.1$ & $<0.1$ & $<0.1$ & 13.2 & $<0.1$ \\
\hline $\mathbf{H}$ & $<0.1$ & 0.8 & $<0.1$ & 0.6 & $<0.1$ & $<0.1$ \\
\hline $\mathbf{I}$ & $<0.1$ & 23.2 & $<0.1$ & $<0.1$ & $<0.1$ & $<0.1$ \\
\hline $\mathbf{L}$ & $<0.1$ & 1.2 & $<0.1$ & $<0.1$ & $<0.1$ & $<0.1$ \\
\hline $\mathbf{M}$ & 19.7 & 54 & $<0.1$ & $<0.1$ & $<0.1$ & 96.2 \\
\hline $\mathbf{N}$ & $<0.1$ & 10.9 & $<0.1$ & $<0.1$ & $<0.1$ & $<0.1$ \\
\hline $\mathbf{Q}$ & $<0.1$ & 7.6 & $<0.1$ & $<0.1$ & $<0.1$ & $<0.1$ \\
\hline $\mathbf{R}$ & $<0.1$ & $<0.1$ & $<0.1$ & $<0.1$ & $<0.1$ & $<0.1$ \\
\hline $\mathbf{S}$ & $<0.1$ & $<0.1$ & $<0.1$ & $<0.1$ & 3.4 & 0.7 \\
\hline $\mathbf{T}$ & $<0.1$ & $<0.1$ & $<0.1$ & $<0.1$ & 0.2 & 3.1 \\
\hline $\mathbf{V}$ & $<0.1$ & $<0.1$ & $<0.1$ & $<0.1$ & $<0.1$ & $<0.1$ \\
\hline $\mathbf{W}$ & $<0.1$ & $<0.1$ & $>99.9$ & $<0.1$ & $<0.1$ & $<0.1$ \\
\hline $\mathbf{Y}$ & 45.4 & $<0.1$ & $<0.1$ & $<0.1$ & $<0.1$ & $<0.1$ \\
\hline
\end{tabular}


Table S3. Position probability matrix of GLU chemical state

\begin{tabular}{|c|c|c|c|c|c|c|}
\hline & $\mathbf{3 1}$ & $\mathbf{1 0 9}$ & $\mathbf{1 2 6}$ & $\mathbf{1 2 9}$ & $\mathbf{1 9 6}$ & $\mathbf{2 5 8}$ \\
\hline $\mathbf{A}$ & $<0.1$ & $<0.1$ & $<0.1$ & $<0.1$ & 3.1 & $<0.1$ \\
\hline $\mathbf{C}$ & $<0.1$ & 0.4 & $<0.1$ & $<0.1$ & 88.9 & 0.8 \\
\hline $\mathbf{D}$ & $<0.1$ & $<0.1$ & $<0.1$ & $<0.1$ & $<0.1$ & $<0.1$ \\
\hline $\mathbf{E}$ & $<0.1$ & $<0.1$ & $<0.1$ & $<0.1$ & $<0.1$ & $<0.1$ \\
\hline $\mathbf{F}$ & 13.6 & $<0.1$ & $<0.1$ & 40.3 & $<0.1$ & $<0.1$ \\
\hline $\mathbf{G}$ & $<0.1$ & $<0.1$ & $<0.1$ & $<0.1$ & 4.5 & $<0.1$ \\
\hline $\mathbf{H}$ & $<0.1$ & 1.6 & $<0.1$ & 0.1 & $<0.1$ & $<0.1$ \\
\hline $\mathbf{I}$ & $<0.1$ & 1.5 & $<0.1$ & $<0.1$ & $<0.1$ & $<0.1$ \\
\hline $\mathbf{L}$ & $<0.1$ & 0.1 & $<0.1$ & $<0.1$ & $<0.1$ & $<0.1$ \\
\hline $\mathbf{M}$ & 39.3 & 0.9 & $<0.1$ & $<0.1$ & $<0.1$ & 3.9 \\
\hline $\mathbf{N}$ & 0.2 & 95.1 & $<0.1$ & $<0.1$ & $<0.1$ & $<0.1$ \\
\hline $\mathbf{Q}$ & $<0.1$ & 0.2 & $<0.1$ & $<0.1$ & $<0.1$ & $<0.1$ \\
\hline $\mathbf{R}$ & $<0.1$ & $<0.1$ & $<0.1$ & 59.6 & $<0.1$ & $<0.1$ \\
\hline $\mathbf{S}$ & $<0.1$ & $<0.1$ & $<0.1$ & $<0.1$ & 1.7 & 76.1 \\
\hline $\mathbf{T}$ & $<0.1$ & $<0.1$ & $<0.1$ & $<0.1$ & 1.8 & 19.2 \\
\hline $\mathbf{V}$ & $<0.1$ & $<0.1$ & $<0.1$ & $<0.1$ & $<0.1$ & $<0.1$ \\
\hline $\mathbf{W}$ & $<0.1$ & $<0.1$ & $>99.9$ & $<0.1$ & $<0.1$ & $<0.1$ \\
\hline $\mathbf{Y}$ & 46.8 & $<0.1$ & $<0.1$ & $<0.1$ & $<0.1$ & $<0.1$ \\
\hline
\end{tabular}


Table S4. Position probability matrix of PLP chemical state

\begin{tabular}{|c|c|c|c|c|c|c|}
\hline & 31 & $\mathbf{1 0 9}$ & $\mathbf{1 2 6}$ & $\mathbf{1 2 9}$ & $\mathbf{1 9 6}$ & $\mathbf{2 5 8}$ \\
\hline $\mathbf{A}$ & $<0.1$ & $<0.1$ & $<0.1$ & 0.2 & $<0.1$ & $<0.1$ \\
\hline $\mathbf{C}$ & $<0.1$ & $<0.1$ & 0.3 & 0.8 & $<0.1$ & $<0.1$ \\
\hline $\mathbf{D}$ & $<0.1$ & $<0.1$ & $<0.1$ & $<0.1$ & $<0.1$ & $<0.1$ \\
\hline $\mathbf{E}$ & $<0.1$ & $<0.1$ & $<0.1$ & $<0.1$ & $<0.1$ & $<0.1$ \\
\hline $\mathbf{F}$ & 57.4 & $<0.1$ & $<0.1$ & $<0.1$ & $<0.1$ & $<0.1$ \\
\hline $\mathbf{G}$ & $<0.1$ & $<0.1$ & $<0.1$ & $<0.1$ & $<0.1$ & $<0.1$ \\
\hline $\mathbf{H}$ & $<0.1$ & $<0.1$ & $<0.1$ & 0.1 & $>99.9$ & $<0.1$ \\
\hline $\mathbf{I}$ & $<0.1$ & 27.2 & $<0.1$ & $<0.1$ & $<0.1$ & $<0.1$ \\
\hline $\mathbf{L}$ & $<0.1$ & $<0.1$ & $<0.1$ & $<0.1$ & $<0.1$ & $<0.1$ \\
\hline $\mathbf{M}$ & $<0.1$ & 3.8 & $<0.1$ & 0.4 & $<0.1$ & $<0.1$ \\
\hline $\mathbf{N}$ & $<0.1$ & $<0.1$ & $<0.1$ & 0.5 & $<0.1$ & 86.9 \\
\hline $\mathbf{Q}$ & $<0.1$ & 0.6 & $<0.1$ & 64.9 & $<0.1$ & 12.8 \\
\hline $\mathbf{R}$ & $<0.1$ & 0.3 & $<0.1$ & 2.5 & $<0.1$ & $<0.1$ \\
\hline $\mathbf{S}$ & $<0.1$ & 0.6 & 0.2 & 30.6 & $<0.1$ & 0.3 \\
\hline $\mathbf{T}$ & 0.2 & 37.1 & $<0.1$ & $<0.1$ & $<0.1$ & $<0.1$ \\
\hline $\mathbf{V}$ & $<0.1$ & 30.2 & $<0.1$ & $<0.1$ & $<0.1$ & $<0.1$ \\
\hline $\mathbf{W}$ & $<0.1$ & $<0.1$ & 99.3 & $<0.1$ & $<0.1$ & $<0.1$ \\
\hline $\mathbf{Y}$ & 42.4 & $<0.1$ & $<0.1$ & $<0.1$ & $<0.1$ & $<0.1$ \\
\hline
\end{tabular}


Table S5. Position probability matrix of HisGluPLP multichemical state

\begin{tabular}{|c|c|c|c|c|c|c|}
\hline & $\mathbf{3 1}$ & $\mathbf{1 0 9}$ & $\mathbf{1 2 6}$ & $\mathbf{1 2 9}$ & $\mathbf{1 9 6}$ & $\mathbf{2 5 8}$ \\
\hline $\mathbf{A}$ & $<0.1$ & $<0.1$ & $<0.1$ & $<0.1$ & 3.6 & $<0.1$ \\
\hline $\mathbf{C}$ & $<0.1$ & 0.5 & $<0.1$ & $<0.1$ & 55.3 & 0.8 \\
\hline $\mathbf{D}$ & $<0.1$ & $<0.1$ & $<0.1$ & $<0.1$ & $<0.1$ & $<0.1$ \\
\hline $\mathbf{E}$ & $<0.1$ & $<0.1$ & $<0.1$ & $<0.1$ & $<0.1$ & $<0.1$ \\
\hline $\mathbf{F}$ & 26.3 & $<0.1$ & $<0.1$ & 52.4 & $<0.1$ & $<0.1$ \\
\hline $\mathbf{G}$ & $<0.1$ & $<0.1$ & $<0.1$ & $<0.1$ & 14.4 & $<0.1$ \\
\hline $\mathbf{H}$ & $<0.1$ & $<0.1$ & $<0.1$ & 1.5 & $<0.1$ & $<0.1$ \\
\hline $\mathbf{I}$ & $<0.1$ & 26.9 & $<0.1$ & $<0.1$ & $<0.1$ & $<0.1$ \\
\hline $\mathbf{L}$ & $<0.1$ & $<0.1$ & $<0.1$ & $<0.1$ & $<0.1$ & $<0.1$ \\
\hline $\mathbf{M}$ & $<0.1$ & 22.2 & $<0.1$ & $<0.1$ & $<0.1$ & 16.7 \\
\hline $\mathbf{N}$ & $<0.1$ & 9 & $<0.1$ & 4 & 1.2 & 1 \\
\hline $\mathbf{Q}$ & $<0.1$ & 39.3 & $<0.1$ & 1 & $<0.1$ & $<0.1$ \\
\hline $\mathbf{R}$ & $<0.1$ & $<0.1$ & $<0.1$ & 29 & $<0.1$ & $<0.1$ \\
\hline $\mathbf{S}$ & $<0.1$ & $<0.1$ & $<0.1$ & 12.1 & 22.7 & 79.2 \\
\hline $\mathbf{T}$ & $<0.1$ & 0.7 & $<0.1$ & $<0.1$ & 2.8 & 2.2 \\
\hline $\mathbf{V}$ & $<0.1$ & 1.1 & $<0.1$ & $<0.1$ & $<0.1$ & $<0.1$ \\
\hline $\mathbf{W}$ & $<0.1$ & $<0.1$ & $>99.9$ & $<0.1$ & $<0.1$ & $<0.1$ \\
\hline $\mathbf{Y}$ & 73.6 & $<0.1$ & $<0.1$ & $<0.1$ & $<0.1$ & $<0.1$ \\
\hline
\end{tabular}


Table S6. Position probability matrix of LEU chemical state

\begin{tabular}{|c|c|c|c|c|c|c|}
\hline & $\mathbf{3 1}$ & $\mathbf{1 0 9}$ & $\mathbf{1 2 6}$ & $\mathbf{1 2 9}$ & $\mathbf{1 9 6}$ & $\mathbf{2 5 8}$ \\
\hline $\mathbf{A}$ & $<0.1$ & 0.6 & $<0.1$ & $<0.1$ & 1.5 & $<0.1$ \\
\hline $\mathbf{C}$ & $<0.1$ & 5.3 & $<0.1$ & $<0.1$ & 0.2 & $<0.1$ \\
\hline $\mathbf{D}$ & $<0.1$ & $<0.1$ & $<0.1$ & $<0.1$ & $<0.1$ & $<0.1$ \\
\hline $\mathbf{E}$ & $<0.1$ & $<0.1$ & $<0.1$ & $<0.1$ & $<0.1$ & $<0.1$ \\
\hline $\mathbf{F}$ & 13.1 & $<0.1$ & $<0.1$ & 92.8 & $<0.1$ & $<0.1$ \\
\hline $\mathbf{G}$ & $<0.1$ & $<0.1$ & $<0.1$ & $<0.1$ & 97.8 & $<0.1$ \\
\hline $\mathbf{H}$ & $<0.1$ & $<0.1$ & $<0.1$ & 5.4 & $<0.1$ & $<0.1$ \\
\hline $\mathbf{I}$ & $<0.1$ & 3.5 & $<0.1$ & $<0.1$ & $<0.1$ & $<0.1$ \\
\hline $\mathbf{L}$ & $<0.1$ & 2.9 & $<0.1$ & $<0.1$ & $<0.1$ & $<0.1$ \\
\hline $\mathbf{M}$ & 29.3 & 2 & $<0.1$ & $<0.1$ & $<0.1$ & $<0.1$ \\
\hline $\mathbf{N}$ & $<0.1$ & 84.4 & $<0.1$ & 0.6 & $<0.1$ & $<0.1$ \\
\hline $\mathbf{Q}$ & $<0.1$ & 1.1 & 9 & $<0.1$ & $<0.1$ & $<0.1$ \\
\hline $\mathbf{R}$ & $<0.1$ & $<0.1$ & $<0.1$ & $<0.1$ & $<0.1$ & $<0.1$ \\
\hline $\mathbf{S}$ & 0.5 & $<0.1$ & $<0.1$ & 1.1 & 0.6 & 98.5 \\
\hline $\mathbf{T}$ & $<0.1$ & $<0.1$ & $<0.1$ & $<0.1$ & $<0.1$ & 1.5 \\
\hline $\mathbf{V}$ & $<0.1$ & 0.2 & $<0.1$ & $<0.1$ & $<0.1$ & $<0.1$ \\
\hline $\mathbf{W}$ & $<0.1$ & $<0.1$ & 90.9 & $<0.1$ & $<0.1$ & $<0.1$ \\
\hline $\mathbf{Y}$ & 57.1 & $<0.1$ & $<0.1$ & 0.1 & $<0.1$ & $<0.1$ \\
\hline
\end{tabular}


Table S7. Position probability matrix of LeuGluPLP multichemical state

\begin{tabular}{|c|c|c|c|c|c|c|}
\hline & $\mathbf{3 1}$ & $\mathbf{1 0 9}$ & $\mathbf{1 2 6}$ & $\mathbf{1 2 9}$ & $\mathbf{1 9 6}$ & $\mathbf{2 5 8}$ \\
\hline $\mathbf{A}$ & $<0.1$ & 0.5 & $<0.1$ & $<0.1$ & 4.4 & $<0.1$ \\
\hline $\mathbf{C}$ & $<0.1$ & 3.9 & $<0.1$ & $<0.1$ & 17.9 & 0.5 \\
\hline $\mathbf{D}$ & $<0.1$ & $<0.1$ & $<0.1$ & $<0.1$ & $<0.1$ & $<0.1$ \\
\hline $\mathbf{E}$ & $<0.1$ & $<0.1$ & $<0.1$ & $<0.1$ & $<0.1$ & $<0.1$ \\
\hline $\mathbf{F}$ & 21.6 & $<0.1$ & $<0.1$ & 29 & $<0.1$ & $<0.1$ \\
\hline $\mathbf{G}$ & $<0.1$ & $<0.1$ & $<0.1$ & $<0.1$ & 49.7 & $<0.1$ \\
\hline $\mathbf{H}$ & $<0.1$ & $<0.1$ & $<0.1$ & 3.1 & 0.2 & $<0.1$ \\
\hline $\mathbf{I}$ & $<0.1$ & 49.2 & $<0.1$ & $<0.1$ & $<0.1$ & $<0.1$ \\
\hline $\mathbf{L}$ & $<0.1$ & $<0.1$ & $<0.1$ & $<0.1$ & $<0.1$ & $<0.1$ \\
\hline $\mathbf{M}$ & $<0.1$ & 6.7 & $<0.1$ & $<0.1$ & $<0.1$ & 0.2 \\
\hline $\mathbf{N}$ & $<0.1$ & 28.2 & $<0.1$ & 4.8 & 0.7 & 0.5 \\
\hline $\mathbf{Q}$ & $<0.1$ & 3.2 & 0.3 & 0.4 & $<0.1$ & $<0.1$ \\
\hline $\mathbf{R}$ & $<0.1$ & 0.4 & $<0.1$ & 32.5 & $<0.1$ & $<0.1$ \\
\hline $\mathbf{S}$ & 0.2 & 0.3 & $<0.1$ & 30.2 & 26.4 & 98.5 \\
\hline $\mathbf{T}$ & $<0.1$ & 2.8 & $<0.1$ & $<0.1$ & 0.7 & 0.3 \\
\hline $\mathbf{V}$ & $<0.1$ & 4.6 & $<0.1$ & $<0.1$ & $<0.1$ & $<0.1$ \\
\hline $\mathbf{W}$ & $<0.1$ & $<0.1$ & 99.7 & $<0.1$ & $<0.1$ & $<0.1$ \\
\hline $\mathbf{Y}$ & 78.1 & $<0.1$ & $<0.1$ & $<0.1$ & $<0.1$ & $<0.1$ \\
\hline
\end{tabular}


Table S8. Position probability matrix of THR chemical state

\begin{tabular}{|c|c|c|c|c|c|c|}
\hline & $\mathbf{3 1}$ & $\mathbf{1 0 9}$ & $\mathbf{1 2 6}$ & $\mathbf{1 2 9}$ & $\mathbf{1 9 6}$ & $\mathbf{2 5 8}$ \\
\hline $\mathbf{A}$ & $<0.1$ & $<0.1$ & $<0.1$ & $<0.1$ & $<0.1$ & $<0.1$ \\
\hline $\mathbf{C}$ & $<0.1$ & 0.4 & $<0.1$ & $<0.1$ & $<0.1$ & $<0.1$ \\
\hline $\mathbf{D}$ & $<0.1$ & $<0.1$ & $<0.1$ & $<0.1$ & $<0.1$ & $<0.1$ \\
\hline $\mathbf{E}$ & $<0.1$ & $<0.1$ & $<0.1$ & $<0.1$ & $<0.1$ & $<0.1$ \\
\hline $\mathbf{F}$ & 0.2 & $<0.1$ & $<0.1$ & 76.9 & $<0.1$ & $<0.1$ \\
\hline $\mathbf{G}$ & $<0.1$ & $<0.1$ & $<0.1$ & $<0.1$ & $>99.9$ & $<0.1$ \\
\hline $\mathbf{H}$ & $<0.1$ & $<0.1$ & $<0.1$ & 18 & $<0.1$ & $<0.1$ \\
\hline $\mathbf{I}$ & $<0.1$ & 2.8 & $<0.1$ & $<0.1$ & $<0.1$ & $<0.1$ \\
\hline $\mathbf{L}$ & $<0.1$ & 0.1 & $<0.1$ & $<0.1$ & $<0.1$ & $<0.1$ \\
\hline $\mathbf{M}$ & 1.1 & 15.3 & $<0.1$ & $<0.1$ & $<0.1$ & 16.1 \\
\hline $\mathbf{N}$ & $<0.1$ & 33.9 & $<0.1$ & 0.6 & $<0.1$ & $<0.1$ \\
\hline $\mathbf{Q}$ & $<0.1$ & 47.4 & 0.5 & $<0.1$ & $<0.1$ & $<0.1$ \\
\hline $\mathbf{R}$ & $<0.1$ & $<0.1$ & $<0.1$ & 4.4 & $<0.1$ & $<0.1$ \\
\hline $\mathbf{S}$ & $<0.1$ & $<0.1$ & $<0.1$ & $<0.1$ & $<0.1$ & 11.9 \\
\hline $\mathbf{T}$ & $<0.1$ & $<0.1$ & $<0.1$ & $<0.1$ & $<0.1$ & 71.9 \\
\hline $\mathbf{V}$ & $<0.1$ & $<0.1$ & $<0.1$ & $<0.1$ & $<0.1$ & $<0.1$ \\
\hline $\mathbf{W}$ & $<0.1$ & $<0.1$ & 99.5 & $<0.1$ & $<0.1$ & $<0.1$ \\
\hline $\mathbf{Y}$ & 98.6 & $<0.1$ & $<0.1$ & $<0.1$ & $<0.1$ & $<0.1$ \\
\hline
\end{tabular}


Table S9. Minimum library size required to predict BCAT mutants using a variety of chemical states

\begin{tabular}{lccccccc}
\hline \multicolumn{1}{c}{ Mutant } & His & Glu & PLP & HisGlu & HisPLP & GluPLP & HisGluPLP \\
\hline V109I/G196C & 24 & 375 & $>500$ & 100 & 108 & 375 & 24 \\
V109I & 12 & 75 & 36 & 50 & 20 & 16 & 12 \\
V109I/Y129F & 12 & 75 & 260 & 50 & 50 & 64 & 12 \\
V109M/G196C & 16 & 375 & $>500$ & 36 & 81 & 375 & 32 \\
\hline
\end{tabular}




\section{Supporting References}

1. Davey, J. A.; Chica, R. A., Multistate Computational Protein Design with Backbone Ensembles. Methods Mol. Biol. 2017, 1529, 161-179.

2. Allen, B. D.; Mayo, S. L., An Efficient Algorithm for Multistate Protein Design Based on FASTER. J. Comput. Chem. 2010, 31, 904-916.

3. $\quad$ Leaver-Fay, A.; Jacak, R.; Stranges, P. B.; Kuhlman, B., A Generic Program for Multistate Protein Design. PLoS One 2011, 6, e20937.

4. Okada, K.; Hirotsu, K.; Hayashi, H.; Kagamiyama, H., Structures of Escherichia coli BranchedChain Amino Acid Aminotransferase and Its Complexes with 4-Methylvalerate and 2-Methylleucine: Induced Fit and Substrate Recognition of the Enzyme. Biochemistry 2001, 40, 7453-7463.

5. Inc, C. C. G. Molecular Operating Environment (MOE), 1010 Sherbrooke St. West Suite \#910, Montreal, QC, Canada, H3A 2R7, 2014.09.

6. $\quad$ Limbach, H. H.; Chan-Huot, M.; Sharif, S.; Tolstoy, P. M.; Shenderovich, I. G.; Denisov, G. S.; Toney, M. D., Critical Hydrogen Bonds and Protonation States of Pyridoxal 5'-Phosphate Revealed by NMR. Biochim. Biophys. Acta 2011, 1814, 1426-1437.

7. Eliot, A. C.; Kirsch, J. F., Pyridoxal Phosphate Enzymes: Mechanistic, Structural, and Evolutionary Considerations. Annu. Rev. Biochem. 2004, 73, 383-415.

8. Wang, J.; Cieplak, P.; Kollman, P. A., How Well Does a Restrained Electrostatic Potential (RESP) Model Perform in Calculating Conformational Energies of Organic and Biological Molecules? J. Comput. Chem. 2000, 21, 1049-1074.

9. $\quad$ Phillips, J. C.; Braun, R.; Wang, W.; Gumbart, J.; Tajkhorshid, E.; Villa, E.; Chipot, C.; Skeel, R. D.; Kalé, L.; Schulten, K., Scalable Molecular Dynamics with NAMD. J. Comput. Chem. 2005, 26, 1781-1802.

10. Renfrew, P. D.; Choi, E. J.; Bonneau, R.; Kuhlman, B., Incorporation of Noncanonical Amino Acids into Rosetta and Use in Computational Protein-Peptide Interface Design. PLoS One 2012, 7, e32637.

11. Privett, H. K.; Kiss, G.; Lee, T. M.; Blomberg, R.; Chica, R. A.; Thomas, L. M.; Hilvert, D.; Houk, K. N.; Mayo, S. L., Iterative Approach to Computational Enzyme Design. Proc. Natl. Acad. Sci. U. S. A. 2012, 109, 3790-3795.

12. Chica, R. A.; Moore, M. M.; Allen, B. D.; Mayo, S. L., Generation of Longer Emission Wavelength Red Fluorescent Proteins Using Computationally Designed Libraries. Proc. Natl. Acad. Sci. U. S. A. 2010, 107, 20257-20262.

13. Allen, B. D.; Mayo, S. L., Dramatic Performance Enhancements for the FASTER Optimization Algorithm. J. Comput. Chem. 2006, 27, 1071-1075.

14. Dunbrack, R. L., Jr.; Cohen, F. E., Bayesian Statistical Analysis of Protein Side-Chain Rotamer Preferences. Protein Sci. 1997, 6, 1661-1681.

15. Mayo, S. L.; Olafson, B. D.; Goddard, W. A., Dreiding - a Generic Force-Field for Molecular Simulations. J. Phys. Chem. 1990, 94, 8897-8909.

16. Dahiyat, B. I.; Mayo, S. L., Probing the Role of Packing Specificity in Protein Design. Proc. Natl. Acad. Sci. U. S. A. 1997, 94, 10172-10177.

17. Lazaridis, T.; Karplus, M., Effective Energy Function for Proteins in Solution. Proteins 1999, 35, 133-152.

18. Shortle, D., Propensities, Probabilities, and the Boltzmann Hypothesis. Protein Sci. 2003, 12, 12981302.

19. Allen, B. D.; Nisthal, A.; Mayo, S. L., Experimental Library Screening Demonstrates the Successful Application of Computational Protein Design to Large Structural Ensembles. Proc. Natl. Acad. Sci. U. S. A. 2010, 107, 19838-19843.

20. Heckman, K. L.; Pease, L. R., Gene Splicing and Mutagenesis by PCR-Driven Overlap Extension. Nat. Protoc. 2007, 2, 924-932.

21. Mironov, G. G.; St-Jacques, A. D.; Mungham, A.; Eason, M. G.; Chica, R. A.; Berezovski, M. V., Bioanalysis for Biocatalysis: Multiplexed Capillary Electrophoresis-Mass Spectrometry Assay for Aminotransferase Substrate Discovery and Specificity Profiling. J. Am. Chem. Soc. 2013, 135, 1372813736.

22. Ernst, O.; Zor, T., Linearization of the Bradford Protein Assay. J. Vis. Exp. 2010, e1918. 